\title{
Educational use of Voice Assistants and Smart Speakers
}

\author{
Thanasis Tsourakas, George Terzopoulos* and Stefanos Goumas
}

Department of Management Science and Technology, International Hellenic University, Kavala University Campus, Greece

Received 04 September 2021; Accepted 16 October 2021

\begin{abstract}
Recently, voice assistants have become very popular since smart speakers incorporate them. This technology is very userfriendly since interaction is carried out using natural language. Voice assistants are being used in everyday life and their technology is constantly evolving, providing new services for the users. Since this technology is used in everyday life by many users, perhaps voice assistants can be used as educational tools. However, there are significant privacy concerns and researchers have discovered serious security risks, although there are studies that provide solutions for these issues. In this study, we focus on the educational use of voice assistants in different levels of education starting from primary school to higher education. Although the popularity of voice assistants is high, there are not many studies regarding their educational potential.
\end{abstract}

Keywords: voice assistants, smart speakers, education, privacy, security.

\section{Introduction}

Augmented Reality (AR), Virtual Reality (VR), and voice are redefining digital experiences by reshaping the way we engage with the world. While today we spend much of our time in front of a screen (smartphone, tablet, laptop), soon, digital experiences will not be confined to a screen. Interaction through voice is the basic distinct characteristic of voice assistants. Using technologies such as voice recognition, speech synthesis, and natural language processing, voice assistants offer a variety of services, from answering different kind of questions asked by users, to controlling other smart devices [1]. Popular voice assistants are Apple's Siri, Google's Assistant, Microsoft's Cortana and Amazon's Alexa.

Voice assistants are mostly incorporated in smart speakers. They provide various capabilities such as: answer users' questions and provide information, play music, set reminders or alarms, online shopping, and gaming. Their capabilities can also be extended by using applications known as «skills» for Amazon Alexa and «actions» for Google Assistant. These applications can be developed by independent developers [2]. With the popularity of voice assistants and smart speakers rapidly growing, it is estimated that the global smart speaker market will reach 163 million units in 2021 and the global smart speaker installed base is forecast to reach 640 million by 2024 [3]. Their acceptance and adoption are high due to various factors, such as the affordable cost for consumers and their ability to communicate through natural language [4].

Nowadays, the use of technology in teaching has become the norm. New immersive technologies such as AR and VR, are used to make education engaging. Voice assistants and smart speakers are a new technology and research on how they can be used for educational purposes is still in an early stage. Reports regarding voice assistants and smart speakers are about home use mostly.

This paper presents findings regarding home usage of voice assistants and smart speakers as well as users' perceptions about them. These findings will assist in understanding the way this technology is used at home and will provide input on how home users see them. Since voice assistants are popular and users (adults or children) have positive attitude towards them, this technology may be used as an educational tool. This paper also presents findings regarding the security of voice assistants and smart speakers. Furthermore, educational uses of voice assistants and smart speakers are also examined since there are some cases reported in the literature. Smart speakers are often used as a source of information or as language learning tools in all educational levels, from primary to higher education. However, there are several reports regarding users' privacy concerns, data collection and management by large companies, so it is evident that these issues must be addressed first.

Our specific research questions (RQ) for the study are as follows:

- RQ1: What cases of voice assistants and smart speakers uses in everyday life have been reported so far in the literature, and what are the perceptions of users about them?

- $\quad$ RQ2: What educational uses of voice assistants and smart speakers have been reported so far in the literature and how students see them?

- RQ3: What security issues of voice assistants and smart speakers have been reported in the literature and what is the perception of users about privacy and data collection of this new technology?

The remainder of the paper is organized as follows. In Section 2, the methodology applied to retrieve relevant papers is described. In Section 3, use cases about voice assistants and smart speakers home usage as well as users' 
perception about them, are presented. Section 4 analyzes the educational possibilities of voice assistants and smart speakers in different levels of education, such as primary, secondary and higher education, as well as in different forms of education such as distance education. Section 5 studies the security level provided by voice assistants and privacy issues reported by users. Finally, Section 6 interprets the findings of this study while in Section 7, new areas for future research are recommended.

Compared to a previous work [5] regarding the educational use of voice assistants and smart speakers, this study presents up to date findings, since the scientific field is relatively new, and this technology is continuously evolving. More research papers on this field are now available and the research interest on this topic is rising. Thus, it is evident that a literature review should be performed frequently since advancements on this technology are vast and the number of reports on its educational use are rising.

\section{Methodology}

In order to retrieve sufficient and high-quality papers, the snowball technique as described in [6] was used. This technique has two steps:

- $\quad$ First, perform a search in online libraries such as Google Scholar, IEEE Xplore and Scopus and gather the initial start set of relevant papers. We used the following keywords: "voice assistants", "smart speakers", "amazon echo", "google assistant" and "Alexa". An initial set of papers was obtained.

- Then, for the initial start set of papers, an iterative process through backward and forward snowballing takes place. Backward snowballing uses the references of a paper to identify new papers, while forward snowballing uses papers that cite the paper being examined.

Using this iterating procedure, 55 relevant papers for the specific RQs of this study were found. As mentioned earlier, although voice assistants are very popular, they are considered a new research topic, thus limited research is done in this field.

\section{Voice assistants use cases in everyday life and users' perception}

The popularity of voice assistants is high. Studies have been conducted to investigate the interactions of users with voice assistants. A literature study [7] regarding intelligent personal assistants (IPAs), provides general insights on how people are using IPAs. Since IPAs were introduced in the 1990s. Authors also provide areas of opportunity of IPAs based on their literature review. Voice assistants incorporated in smart speakers are considered IPAs, thus some papers regarding them are also included in the review. Findings suggest that people use voice assistants mainly for simple tasks such as listening to music, searching for information, or getting weather forecasts. Authors also found areas where opportunities can arise from voice assistant's implementation, such as the educational sector, as voice assistants can be used for language learning, and the health care sector, where voice assistants can suggest treatments based on stored information about patients' vital signs.
Data can be collected from voice assistants in order to see how they are used. Ammari et al. [8] has followed this direction, collecting data, nearly 200.000 commands, from logs of two well-known voice assistants, Google Assistant and Amazon Alexa, in order to understand how individuals are using them. The most popular reason why people use Google Assistant is searching of information (26\%) and listening to music $(23.4 \%)$. It is interest to point out that commands that Google Assistant failed to understand was 17\%. In addition to Google Assistant, the most popular reason people are using Amazon Alexa was music (28.5\%) while $11 \%$ of commands were not understood. However, in both cases, there was a form of social interaction with the assistant. Nearly $2 \%$ of interactions were polite compliments to the voice assistant.

Libraries also use voice assistants to provide basic information, according to research conducted by Sweeney and Davies [9], with data collected from 1929 public and academic libraries in United States. Another study also confirms that the most common uses of Amazon Alexa were weather forecasts, searching of information, current news updating, controlling other devices, activities reminders, music, fun, setting timer and checking time [10]. Furthermore, factors that can affect the interaction between people and voice assistants are the duration of possessing the devices and the placement in specific places of the house, while factors such as age and how familiar is this technology to people did not affect the user interaction.

Same findings arose from another study [11], where people aged 65 and above, use voice assistants mainly for searching information, activities reminders, music, and weather forecasts. An equally important users' group who take advantage of voice assistant technology is children. They use them for listening to music and stories, getting information and answers about different topics and play games according to the analysis of a totally 1505 reviews of Amazon Echo Dot Kids device conducted by Radford et al. [12].

Beyond how people use voice assistants, the perceived user experience is also an important issue. User experience from voice assistants in case of home usage was reported in [13]. After the data collection from a total of 75 participants using Amazon Alexa devices and the interviews that took place, most of the participants reported an overall positive user experience and the majority of them had more than one device in their homes. Positive experiences were also confirmed by other studies [14], where users reported the memorable experiences they had from their interaction with voice assistants.

Children feel that voice assistants are friendly and worthy of trust, while they are satisfied with their use at the same time [15]. Elderly people over 65 years opinions differ, since they feel strange to talk to a machine, in their first contact with voice assistants [11]. User experience can also be conceivable from people's evaluations. For example, the collection and analysis of 55.502 reviews of the Amazon Echo smart speaker device from the official product page, proves that this specific device is ideal to help users in various issues and keeps them company [16].

However, besides basic functionality, voice assistants support custom applications from developers. In a study [17] about the user experience perceived, more than 2.800 Amazon Alexa skills were evaluated. Users reported their dissatisfaction with different issues related to the content of a skill, errors where the skill did not respond properly, 
malfunctions after software upgrade and failure of the skill to understand the commands given by users.

Summarizing the findings in this section, related to RQ1, voice assistants are mainly used for simple tasks such as searching of information and playing music. Although the number of studies is limited (11), it appears that users report positive experiences, while in some cases difficulties have been reported in the understanding of commands by voice assistants.

\section{Voice assistants in education and students' experience}

4.1. Artificial Intelligence (AI) and chatbots in education Voice assistants are based on AI and can be considered as chatbots; thus, it is important to investigate the use of AI and chatbots for educational purposes. AI is a technological innovation, the intervention of which has greatly increased in different areas [18]. Although there is no general solution to the application of AI to all education levels, Wong et al. [19] claims that interacting with AI through educational games can be a great starting point. According to the authors, for primary school students aged 7 to 8 , the interaction with AI should follow a game type approach and students should understand how AI has an impact on everyday life and learn to interact with sensors. Slightly older students ( 8 to 12 years old), should begin to build on concepts and ideas they have acquired and create and modify perception-based applications, while older students (12 to 15 years old) should be able to develop high-order computational thinking skills.

According to Pedro et al. [20], AI can be used to ensure fair and inclusive access to education. Authors also claim that education has crucial role in striving for a future ready to embrace AI. Many countries, such as China, Latin America, Brazil, and South Africa have followed this direction, adopting technological solutions for including AI in the curriculum.

The market for AI in education is estimated to grow rapidly between 2018 and 2025 [21]. The North American market is so far a leader in innovation and other technological advantages, which can be attributed in high levels of investments in research in AI, while it turns out that there are different views on the feasibility of using this technology for educational purposes.

However, at the same time, research conducted by Jindal and Bansal [22], refers to the lack of knowledge about AI. In the above study, out of a total 226 medical students, only $141(62.5 \%)$ have some basic knowledge about this subject, with the Internet being the main source of information. It is also interesting the fact that the majority of students (71\%) states that this technology should be included in the medical field.

At this point, both advantages and disadvantages of the application of AI in the education field should be pointed out. According to [20], an observed advantage is the various technology possibilities, such as the robotic telepresence, which allows students with disabilities attend classes from everywhere, thus creating more accessible environments so that teachers can focus on the above students. Another advantage of $\mathrm{AI}$ is the promotion of computational thinking, which has emerged as one of the key skills that enables students to thrive in a society that is oriented towards it. According to [18], in countries such as India, where there is a shortage of educational staff, the application of AI can provide high-quality learning. In this case, teachers' duties can be easier, so that they can focus on teaching quality.

Apart from AI application advantages, there are also challenges. According to [20], a major challenge of AI use in education, can be the fact that not only teachers need to be prepared for AI introduction in education, but also this new technology should be presented in such a way that it is accessible and understandable by students.

Regarding chatbot software, its use has been tested to a limited extent in the education field, mainly in higher education institutes. In most cases, chatbot software is treated simply as a means by which users can ask a question and receive an answer.

Seeber [23], studies whether chatbot software affects students learning behavior and learning outcomes in addition to traditional teaching materials. In the research, participants were high school students aged 14 to 18 years. In conclusion, students demonstrate higher motivation in the learning process using chatbot software, compared to traditional teaching materials, and with a chatbot software that includes game elements (a quiz game), students achieve better learning outcomes.

Finally, for universities, Winkler et al. [24] developed Sara, a chatbot software, that provides services by text and voice. The review by 182 students showed significantly improved understanding during computer programming courses.

\subsection{Voice assistants as language learning tools}

Voice assistant's ability to speak and understand multiple languages can help students with their vocabulary and pronunciation [1]. In many countries such as India, where English language is used as a secondary language to communication, voice assistants can help students learn different languages [25]. In this case, the advantages from voice assistants, and especially Alexa, are their ability to be used individually by each student. Alexa can recognize who is asking the questions and provide personalized responses. As a result, students feel more comfortable during interaction. Another advantage is the dialogue, which helps students develop their speaking skills, as well as language understanding.

Educational applications that can be adapted to voice assistants such as the one developed by Bilic et al. [26], may be useful in the educational process. The application with the name Austria and Europe Game, based on Alexa, was developed at the Graz University of Technology, and its target audience is people who speak both German and English language. The evaluation of the interactive game by a sample of 2 teachers and a total of 16 students showed that people who interacted with the application were quite satisfied, despite several failures such as the vocabulary misunderstanding by Alexa.

Moussalli and Cardoso [27] conducted an experiment, in which 11 students (aged 19 to 30 years old) who spoke different languages participated, and the goal was to learn English language. The results of this experiment with the Amazon Echo smart speaker, prove that Alexa can understand students and the opposite. Specifically, students were able to understand Alexa with an accuracy of more than $80 \%$. Of the approximately 1.000 interactions that users had with the Echo device, 177 failed, due to the pronunciation of words in English language.

In another research [28] aimed to study the effectiveness of voice assistants, and specifically Google Assistant in language learning, data collected from a sample of 10 
students in a Malaysia area, showed that Google Assistant is a functional tool that can be applied in teaching and especially in language learning.

Dizon [29] also studied the adequacy of voice assistants in language learning, with an experiment of 4 Japanese students in order to learn English. The results of the experiment showed that at first Alexa was not able to understand more than $50 \%$ of the students' voice commands. One possible explanation for this is that students' pronunciation was not at an adequate level. Dizon [30] reevaluated Alexa as a language learning tool, with a 15-week experiment in which 28 students participated from a university in Japan. The results showed that Alexa did not have a significant impact on students' listening skills. Students did not completely understand the answers from Alexa due to the rhythm of speech and the level of vocabulary, which made it difficult for them. A possible explanation for this, is that students focused more on speaking English than trying to understand Alexa's answers. In another experiment [31], participants are divided to two groups. The first group consists of people who use English as their main language in order to communicate and the second group with people who use English as a second language. Authors conclude that there is no statistically significant difference in how both groups interact with Siri and Alexa. Furthermore, it appears that the participants of the second group expressed difficulties in understanding words, while in terms of the level of user satisfaction the first group seems to have higher satisfaction rates than the second group.

\subsection{Voice assistants in primary education}

Educational settings such as the one developed by David et al. [32], can offer opportunity to primary school teachers for controlling and managing all of their students. This specific environment includes voice assistants and can recognize students' emotions during the lesson with the help of cameras. Butler [33], examined the involvement of voice assistants and especially Google Assistant, in order to find out how students use voice assistants in two elementary schools at New Zealand. From the data collected by two voice assistants it appears that the questions asked to them by students are divided into three categories a) questions related to a learning object, b) questions about the operation of the voice assistant (about procedure used in order to ask questions), c) questions related to games, curiosity, or local information. Furthermore, students have used voice assistants for calculations, word explanations, and spelling, with teachers point out the positive impact from using them in the classroom environment.

Dousay and Hall [34] also studied the use of the Amazon Echo Dot smart speaker in a classroom environment for primary education. In this case, 900 primary school students used 90 Amazon Echo Dot devices to obtain information and for many other educational activities, such as spelling. Teachers used the device in order to help them in their work. It was reported that the use of voice assistants has been constructive and exciting for students and teachers.

Custom applications for voice assistants can also be useful in the educational process. The application "Bake a cake $1 \mathrm{x} 1$ " developed by Schoegler et al. [35], helps primary school children to further develop their math skills. With the use of this application by students aged 6 to 13 years, their impressions were positive.

\subsection{Voice assistants in secondary education}

In secondary education, teachers describe their students' interaction with voice assistant technology as a positive experience for both themselves and their students [36]. In order to understand the attitudes of high-school students through the programming process of the voice assistant Alexa, Van Van Brummelen [37], conducted an experiment. A total of 47 students participated in this experiment, who during the laboratory part were divided into 2 groups (first group 12 students, second group 35 student). The research question was how programming applications (skills) for Alexa and learning more about voice assistants, affects students' beliefs about AI and Alexa. Comparing the answers from the questionnaires before and after the workshop from the category related to Alexa 'personality', significant differences were found in how students felt about Alexa's intelligence and how familiar Alexa was to them. Students were optimistic about the future of conversational AI and they could think of interesting, positive applications for conversational AI in everyday life.

\subsection{Voice assistants in higher education}

In higher education, the application of voice assistant technology can be a useful solution in many cases. Voice assistants such as Siri, Alexa, Cortana, can offer personalized suggestions [38]. Also, as there are various systems with AI that are increasingly being used in schools, colleges and universities, voice assistant applications, like the one proposed by Serban and Todericiu [2] with the name "Alexa for Uni", can offer services to both students and teachers. Alexa for Uni uses Alexa and can communicate with services such as Microsoft Office. Moreover, through this application, information related to the university can be quickly acquired, while the goal is to facilitate interaction between teachers and students through communication tools. An experiment conducted by Winkler et al. [39], aimed to understand whether the use of voice assistants can improve learning outcomes within a group. From the total of 120 higher education students who participated in the experiment and after being divided into two groups, it appears that the team which interacted with the Alexa voice assistant achieved a higher quality of cooperation and a better result in solving the problem assigned to both groups.

Winkler et al. [40] also studied what higher education students experience from voice assistants as instructors. Students were able to work individually for 30 minutes to solve a complex problem with the help of a voice assistant. Initial results of this experiment show that voice assistants with which users can interact through voice commands, are better than cases where the interaction is based on written commands.

Different interfaces such as the one developed by Bortoli et al. [41] for use by students of Modena and Reggio Emilia University and in particular the Department of Communication and Economics (DCE), may be extremely useful for students to obtain information. The above interface was based on the Alexa voice assistant and aims to improve accessibility to the information available on the department's website. Its operation is simple and is based on the needs of users to search for information about the lesson schedules, exam periods and teachers' office hours. The results of the evaluation by 20 students, show the high percentage of user satisfaction.

Ondáš et al. [42] developed an application-skill aimed at providing information for the Department of Electronics and multimedia telecommunications at the University of 
Slovakia, for Amazon Echo devices. During evaluation of the application through questionnaires, $87 \%$ of students have received the necessary information they wanted. Also, regarding the impressions of students from the interaction with the application, $62.5 \%$ of them, state positive impressions.

Another application is the one developed by OchoaOrihuel et al. [43] based on Alexa. The application works with a course management system (Moodle). Its purpose is to retrieve information from the course management system, a process necessary for the application to communicate with Moodle online services. Based on the evaluation of this application by a group of 61 students, students reported that they did not use the application very often because they felt that it did not provide enough security to offset the potential risks of privacy.

For students on the autism spectrum, an application developed based on Alexa, called MotivateMe [44], aims to help students improving their daily education live. Specifically, the purpose of MotivateMe application is to motivate students in higher education with autism, where the main problem they face is the lack of attention and motivation. In the application, graphic elements are displayed on a screen, which help users stay focused on their goal, while having the ability to perform various tasks such as studying for a specific course.

Another interesting issue is the influence of voice assistants on students. Sáiz-Manzanares et al. [45], conducted a research to answer questions about students learning outcomes from the digital assistants use or non-use of a Learning Management System (LMS) that integrates voice assistants. The data collected from a total of 109 students at Burgos University show that the level of students' satisfaction in teaching using voice assistants was high, while at the same time most students seem to accept the use of voice assistants to provide them information on various topics, with the same students to point out that there is room of improvement.

\subsection{Voice assistants in distance learning}

Regarding online education, Zhao et al. [46] propose a voice assistant-based system that is dialogue-oriented. The system will have a dual role. It can act as a student and interact with other students, and at the same time take the role of a teacher who provides guidance to students in an e-learning environment.

Emerling et al. [47] also propose using voice assistants for distance learning. Teachers can create and add their own applications (skills), in order to help them in their educational work. Authors suggest using the online environment called Blueprint for building skills for Alexa. They also propose the skill Task Tracker, which aims to help teachers stay organized by creating to-do lists.

\subsection{Perceptions about educational use of voice assistants}

Data related to the experience of using voice assistants has also been collected from the education sector. Babic et al. [48] collected data from 309 students who participated in the research, using Google Assistant and Lyra for the first time. The areas where students evaluated these voice assistants were usefulness, user satisfaction and response time. Students reported that they are willing to continue using voice assistants in the future.

With the goal to investigate children's interactions and perceptions of voice assistants, Festerling and Siraj [49] collected data from questionnaires from 27 children aged 6 to 10 years old. The results indicate that children showed great enthusiasm using voice assistants. Children believe that machines remain servants of their users, implying the superiority of people in terms of ethics and intelligence.

Another experiment was conducted by Van Brummelen et al. [50] in order to understand the perceptions of high school students about voice assistants and especially Alexa. Students felt that Alexa is more intelligent than they initially thought.

Teachers' perceptions of voice assistant technology were studied by Incerti [51]. Data was collected from a sample of 68 students (preservice teachers) from Midwestern University, who said without hesitation that they would use the Amazon Echo device in elementary school classrooms. However, they also point out the various difficulties face by the application of this technology in a classroom environment, such as the inaccuracy of voice assistant's answers and the unavailability of wireless networking in the classroom.

Lopatovska and Oropeza [52] studied students' reactions by choosing to place an Amazon Echo device in a public academic environment. The data collected by the device itself indicate that the two most common interactions of students with Alexa, were questions on various topics and polite compliments to the voice assistant. Also, it is noteworthy that most students did not interact with the voice assistant since they did not feel comfortable with the device, which shows the mixed tendencies for the adoption of voice assistants.

Summarizing the findings in this section regarding RQ2, voice assistants have been used at all levels of education for educational purposes. In most cases, positive impressions and a high level of user satisfaction have been reported. The number of relevant studies is limited (24) although the number is constantly rising over the years. In the case of using them as language learning tools, issues of understanding users' commands by voice assistants have been reported, although, most users adjust their pronunciation and vocabulary to be understandable.

\section{Voice assistants' privacy and security}

Lemmer [53] examined whether Amazon Echo and Google Home devices comply the General Data Protection Regulation (GDPR). The study focused on three categories of features: transparency, parental consent under Article 8 of the GDPR, and the right of users to delete data collected by voice assistants. As follows, for the two smart speakers on this issue, no relevant documentation is provided with the company's privacy policy. Also, regarding custom applications (skills/actions), no relevant documentation is provided in order to show how the processing and data sharing takes place. However, there are differences between the two devices regarding parental consent, with Google arguing that the collection of data related to children's conversations is based on the legal framework of parental consent, while Amazon has no specific policy on this issue. In addition, although users can delete the data collected by voice assistants, this process can become quite complicated and misleading.

Privacy is a factor that can affect the decisions of users for acceptance and adoption of voice assistants according to Lau et al. [54]. Specifically, participants who already owned a voice assistant stated that they had set up their devices without the help of a third party, while the factors that affect 
them regarding the acquisition of voice assistants are the convenience which they offer to perform various activities, but also the fact that people want to own this technology earlier than others (early adopters). For these people, convenience is preferred over privacy.

Another survey on the role of privacy in the acceptance and use of digital assistants was conducted by Liao et al. [55]. In this case, out of 1.178 academic staff, it was found that people with a higher level of digital education reported less privacy concerns and more confidence in how their data is used by voice assistants.

In order to measure the effectiveness of privacy policies provided by developers of voice assistant applications, Liao et al. [56] analyzed a total of 64.720 Amazon Alexa skills and 2.201 Google Assistant actions. The results show that there are not privacy policies for all applications. Specifically, for Alexa the percentage is $56.4 \%$ while for Google is $12.2 \%$. Also, for many applications there is the same hyperlink which leads to the same privacy policy. Regarding on how easy is for someone to access privacy policies, there is a difficulty with accessibility, since privacy policies are not directly accessible through devices, and often are long enough so that users do not read them.

Additionally, the use of the female gender in voice assistants can cause social harm from the indirect discrimination of women, as claimed by Loideain and Adams [57]. According to them, technologies based on AI can also reproduce various prejudices that can be embedded in the social fabric. Policy from European Union, American and United Kingdom documents takes all of the above into account and proposes a shift towards ethics as a framework for thinking and dealing with the negative social impact of technologies based on AI.

Manikonda et al. [58], identified seven categories of issues on which users are concerned about the privacy of voice assistants: 1) device hacking, 2) obtaining users' personal data, 3) recording private conversations 4) continuous recording of conversations, 5) respect for the privacy of users, 6) storage of data collected, and 7) use of voice assistant data (e.g., for research purposes).

Pal et al. [59] analyzes the categories of privacy issues faced by users as follows: issues related to the collection of user data by voice assistants (e.g., recording a private conversation), issues related to the recording of data (e.g., a conversation) by persons other than users of voice assistants without their consent, issues related to the sharing of data collected by voice assistants with other services in order to provide some functions (e.g., when the user does not want to share their location while the voice assistant continues to monitor them in order to provide a third party function) and issues related to environmental secrecy (monitoring all user data not only for reasons of national security, but also to improve services).

When it comes to user safety, people are concerned about a variety of issues, most notably the unwanted recording of a conversation by voice assistants. Malkin et al. [60] studied opinions of 116 people who own Amazon and Google voice assistants, and found that half of the participants believe that conversations are stored and used by companies. When asked how do they feel if the recorded conversations are stored for a certain period of time, $90.8 \%$ answered that they feel comfortable if their conversations are kept for a week, although this percentage decreases $(57.7 \%)$ if the storing period is a year.

[61] Cho et al. (2020) surveyed a sample of 90 people from a university in the United States. The results of this research reveal that the adjustment of privacy settings by the users do not affect the trust, reliability, security and usability of voice assistants.

Another research that aims to examine how users perceive the security and privacy of voice assistants was conducted by Chalhoub and Flechais [62]. In this research, data collected from 13 people through interviews, lead to the conclusion that people are not worried about sharing their personal data because they have nothing to hide. Concerning monitoring, participants expressed no concern. Concerns about the recording of their conversations by voice assistants were also dismissed, claiming that voice assistants are no different than smartphones. Therefore, in this research as well others, users sacrifice privacy for the convenience provided by voice assistants.

Lei et al. [63], in an attempt to highlight the vulnerabilities of voice assistants, examined Alexa. The first vulnerability identified is the certification by which the user can access the voice assistant. In the case of the Amazon Alexa the word of activation or otherwise "Wake Word" is the word 'Alexa'. Although the user has the option to change the activation word, the alternatives are limited to the words 'Amazon', 'Echo', 'Computer'. A second vulnerability is the fact that the voice assistant can accept commands even if there is no one nearby, as long as the physical presence of the person who wishes to interact with it is not required. The third vulnerability is that Alexa's owners can control the various smart devices that are connected to the network with commands that include the device name (which can be modified, but usually users don't change it) followed by the command.

Another study to highlight security issues of voice assistants was conducted by Castell-Uroz et al. [64], who analyzed the servers and services that Amazon Alexa has daily access. From this process, it emerged that when a voice assistant communicates with a service at the user's command and uses the http protocol, it is not difficult for someone to access the data sent to Amazon servers.

Sharif and Tenbergen [65], also conducted a literature study to identify the following vulnerabilities in voice assistants: 1) their characteristic element is that they always hear everything from the environment in which they are located, 2) weak authentication, 3) attacks which occur due to weak authentication, 4) vulnerabilities in the cloud infrastructure on which they rely, 5) vulnerabilities due to the ability of users to upgrade the functionality of voice assistants with applications that automate a particular task, 6) vulnerabilities which are observed when interconnecting other devices with voice assistants where someone can take advantage to gain access to voice assistants or even spy on users.

Furthermore, according to Chung et al. [66], the categories of attacks that can take place on voice assistants are a) Distributed Denial of Service (DDoS) attacks, b) malicious voice commands that can be given by a pretender of the owner of the voice assistant, c) various voice stimuli which can be recorded within the range of the voice assistant and possibly sent to a server, d) the recording and interception of data circulating on the network (sniffing attack).

As with voice assistants, vulnerabilities are now identified in custom applications that are part of this technology. Zhang et al. [67] identify the problem that a malicious user can develop and publish an application with a similar name to another application aiming of deceiving users. As a result, malicious users can obtain information 
about the owners of voice assistants.

But despite the vulnerabilities in digital assistive technology, there are countermeasures reported in the literature, that users can take to protect themselves. In order to protect users from unauthorized access to a voice assistant using voice commands, Feng et al. [68] developed and proposed a system which offers continuous authentication. This system called VAuth, is designed to be applied to devices that people usually carry with them, for example smartwatches. The purpose is to ensure that the recorded voice commands come directly from the user. VAuth evaluation on different scenarios and speakers proves that it achieves more than 95\% accuracy in recognizing voice commands to voice assistants, and when a user does not speak, it prevents any unauthorized access to the voice assistant.

Sharif and Tenbergen [65] also refer to the measures that users can take, in order to protect themselves from the vulnerabilities of voice assistants. They suggest turning off the digital assistant microphone when not in use. Alhadlaq et al. [69] refers also to the issue of protecting users from the vulnerabilities of voice assistants. According to them, Amazon offers privacy options related to sound, the unique identity of a user (User ID) and the location of the users. Specifically, when users interact with applications using voice commands, audio is not accessible to developers. Instead, all voice commands are transcribed and sent to developers as text. This design option from Amazon has many benefits of privacy: a) developers can't create one unique user footprint using rich audio data and b) voice data can't be used to infer age, gender, etc. Regarding the unique user ID (User ID), Amazon uses for each application-skill a unique identity of each user (User ID). Finally, in terms of user location, developers can configure applications to request access to the user's location (country or zip code only).

Summarizing the findings in this section regarding RQ3, voice assistants have several issues that need to be addressed so that users feel comfortable using them. Although many users do not care about privacy of their data and show blind trust in companies, there are many who are still skeptical. Research shows that there are still several vulnerabilities that need to be addressed in order voice assistants to be used as educational tools.

\section{Discussion}

Voice assistants is a popular technology. They are used in many houses by children, young people and elderly people. Regarding RQ1, from 7 studies found in the literature, it is concluded that voice assistants are mainly used to perform simple tasks such as playing music, search information and other similar processes. Generally, users report positive experiences about using them, although there have been cases where difficulty in understanding commands was reported. Young children are trying to be understood by voice assistants and adjust their strategy when asking questions. This eventually will assist them to speak more fluently and to create more correct sentences.

Regarding RQ2, in educational field, voice assistants have been used at all levels of education. In general, as shown by 9 studies in the literature, AI and chatbots has been used in education and there is potential for both $\mathrm{AI}$ and chatbots. However, teacher training is considered necessary, and the technology should be presented in an attractive and understandable way to students. Regarding language learning, findings from 7 research papers reveal early attempts of using voice assistants as language learning tools. As a result, there is potential, although for users that have low level of understanding and speaking, voice assistants are not trying to adapt and understand them or simplify their vocabulary when responding to questions. This may be a significant drawback when young children are using them, especially for foreign language learning.

A small number of studies (4), report results regarding primary education. Elementary students and teachers enjoy interactions with voice assistants in a classroom environment, have positive impressions and show enthusiasm. The same conclusions came out from 2 other studies in secondary education. In higher education, 9 studies were located. Results show that voice assistants, when used, can achieve a higher quality of cooperation and a better result in problem solving. Custom applications developed for providing information to students show high percentage of user satisfaction although there are privacy issues that discourage users from using them more often. Two papers also suggest ways for voice assistants to be used in distance learning. Students and teachers see the potential of this technology, although they feel that there is room for improving their services.

Regarding RQ3, there are significant privacy issues reported by the users. In total, 17 papers were located addressing privacy and security issues. Voice assistants have several security issues (such as poor authentication), that need to be addressed in order for users to feel more comfortable while using them. Although many users don't care about their data privacy, there are also many who are still more skeptical using this technology. On the other hand, there are some security countermeasures that users can take in order to improve security.

\section{Conclusion}

Immersive technologies such as Augmented Reality, Virtual Reality and voice assistants, will shape education in the near future. Voice assistants as an innovative and rapidly evolving technology, can be used in all areas of people's daily lives and especially in the educational field. In this paper, 55 studies were located regarding voice assistants everyday use, their educational potential and issues regarding privacy and security that need to be addressed. Although the number of studies regarding voice assistants is relatively low, there is significant research interest on this new technology. Nearly half of the papers presented are from 2020. Besides privacy and security challenges that need to be addressed, voice assistants do not speak all languages and this may limit their use in an educational setting. Furthermore, besides students, teachers should be trained and motivated to use this technology in the classroom.

This is an Open Access article distributed under the terms of the Creative Commons Attribution License. 


\section{References}

1. Terzopoulos, G., \& Satratzemi, M. (2020). Voice Assistants and Smart Speakers in Everyday Life and in Education. Informatics in Education, 19(3), 473-490. DOI:10.15388/infedu.2020.21.

2. Şerban, C., \& Todericiu, I. A. (2020). Alexa, What classes do I have today? The use of Artificial Intelligence via Smart Speakers in Education. Procedia Computer Science, 176, 2849-2857. DOI: 10.1016/j.procs.2020.09.269.

3. Canalys. (2020). Canalys: Global smart speaker market set to reach 163 million units in 2021. Retrieved July 22, 2021 from https://canalys-com-public-prod.s3.eu-west2.amazonaws.com/static/press release/2020/SSPR2020Q2.pdf.

4. Burbach, L., Halbach, P., Plettenberg, N., Nakayama, J., Ziefle, M., \& Valdez, A. C. (2019). "Hey, Siri"," Ok, Google"," Alexa". Acceptance-Relevant Factors of Virtual Voice-Assistants. In: 2019 IEEE International Professional Communication Conference (ProComm), pp. 101-111. DOI: 10.1109/ProComm.2019.00025.

5. G. Terzopoulos and M. Satratzemi, "Voice Assistants and Smart Speakers in Everyday Life and in Education," Informatics in Education, pp. 473-490, Sep. 2020, doi: 10.15388/infedu.2020.21.

6. Wohlin, C. (2014). Guidelines for snowballing in systematic literature studies and a replication in software engineering. In: Proceedings of the 18th International Conference on Evaluation and Assessment in Software Engineering (EASE '14), pp. 1-10. DOI: http://dx.doi.org/10.1145/2601248.2601268.

7. de Barcelos Silva, A., Gomes, M. M., da Costa, C. A., da Rosa Righi, R., Barbosa, J. L. V., Pessin, G., ... \& Federizzi, G. (2020). Intelligent personal assistants: A systematic literature review. Expert Systems with Applications, $147 . \quad$ DOI: 10.1016/j.eswa.2020.113193.

8. Ammari, T., Kaye, J., Tsai, J. Y., \& Bentley, F. (2019). Music, Search, and IoT: How People (Really) Use Voice Assistants. $A C M$ Trans. Comput. Hum. Interact., 26(3), 1-28. DOI: $10.1145 / 3311956$.

9. Sweeney, M. E., Davis, E. (2020). Alexa, are you listening? An exploration of smart voice assistant use and privacy in libraries. Information Technology and Libraries, 39(4), 1-21. DOI: 10.6017/ital.v39i4.12363.

10. Lopatovska, I., Rink, K., Knight, I., Raines, K., Cosenza, K., Williams, H., Sorsche, P., Hirsch, D., Li, Q., Martinez, A. (2019). Talk to me: Exploring user interactions with the Amazon Alexa. Journal of Librarianship and Information Science, 51(4), 984-997. DOI: $10.1177 / 0961000618759414$

11. Pradhan, A., Lazar, A., \& Findlater, L. (2020). Use of intelligent voice assistants by older adults with low technology use. ACM Transactions on Computer-Human Interaction (TOCHI), 27(4), 127. DOI: $10.1145 / 3373759$.

12. Radford, M., Floegel, D., Barriage, S., \& Houli, D. (2019). “Alexa, where do babies come from?" Investigating children's practices with intelligent personal assistants. In: ALISE 2019 Conference Proceedings.

13. Sciuto, A., Saini, A., Forlizzi, J., Hong, J. I. (2018). Hey Alexa, What's Up?: A mixed-methods studies of in-home conversational agent usage. In: Proceedings of the 2018 Designing Interactive Systems Conference, pp. 857-868. DOI: 10.1145/3196709.3196772.

14. Lopatovska, I., \& Williams, H. (2018). Personification of the Amazon Alexa: BFF or a mindless companion. In: Proceedings of the 2018 Conference on Human Information Interaction \& Retrieval, pp. 265-268. DOI: 10.1145/3176349.3176868.

15. Druga, S., Williams, R., Breazeal, C., Resnick, M. (2017). Hey Google is it $\mathrm{OK}$ if I eat you?: Initial explorations in child-agent interaction. In: Proceedings of the 2017 Conference on Interaction Design and Children, pp. 595-600. DOI: 10.1145/3078072.3084330.

16. Gao, Y., Pan, Z., Wang, H., \& Chen, G. (2018). Alexa, my love: Analyzing reviews of amazon echo. In 2018 IEEE SmartWorld, Ubiquitous Intelligence \& Computing, Advanced \& Trusted Computing, Scalable Computing \& Communications, Cloud \& Big Data Computing, Internet of People and Smart City Innovation (SmartWorld/SCALCOM/UIC/ATC/CBDCom/IOP/SCI), pp. 372380, DOI: 10.1109/SmartWorld.2018.00094.

17. Atefi, S., Truelove, A., Rheinschmitt, M., Almeida, E., Ahmed, I., \& Alipour, A. (2020). Examining user reviews of conversational systems: a case study of Alexa skills. arXiv preprint
arXiv:2003.00919.

18. Kadam, P. V. (2019). Artificial Intelligence and Innovations in Education. International Journal of Scientific Development and Research, 4(10), 169-174.

19. Wong, G. K., Ma, X., Dillenbourg, P., \& Huan, J. (2020). Broadening artificial intelligence education in K-12: where to start?. ACM Inroads, 11(1), 20-29. DOI: 10.1145/3381884.

20. Pedro, F., Subosa, M., Rivas, A., \& Valverde, P. (2019). Artificial intelligence in education: Challenges and opportunities for sustainable development.

21. Osetskyi, V., Vitrenko, A., Tatomyr, I., Bilan, S., \& Hirnyk, Y. (2020). Artificial intelligence application in education: financial implications and prospects. In: Financial and credit activity: problems of theory and practice, pp. 574-584. DOI:10.18371/fcaptp.v2i33.207246.

22. Jindal, A., \& Bansal, M. (2020). Knowledge and education about artificial intelligence among medical students from teaching institutions of India: a brief survey. MedEdPublish, 9 . DOI:10.15694/mep.2020.000200.1

23. Seeber, I. (2019). Chatbots in Education (Doctoral dissertation).

24. Winkler, R., Hobert, S., Salovaara, A., Söllner, M., Leimeister, J. M. (2020). Sara, the lecturer: Improving learning in online education with a scaffolding-based conversational agent. In: Proceedings of the 2020 CHI Conference on Human Factors in Computing Systems, pp. 1-14. DOI: 10.1145/3313831.3376781.

25. Sandeep, S. S. (2019). Learning English language through Amazon Alexa for Indian students. Journal of the Gujarat Research Society, 21(10), 619-622.

26. Bilic, L., Ebner, M., \& Ebner, M. (2020). A Voice-Enabled Game Based Learning Application using Amazon's Echo with Alexa Voice Service. In: International Journal of Interactive Mobile Technologies (iJIM), pp. 226-232. DOI:10.3991/ijim.v14i03.12311.

27. Moussalli, S., \& Cardoso, W. (2020). Intelligent personal assistants: can they understand and be understood by accented L2 learners?. Computer Assisted Language Learning, 33(8), 865-890. DOI:10.1080/09588221.2019.1595664.

28. Sing, P. B., Embi, M. A., \& Hashim, H. (2019). Ask the Assistant: Using Google Assistant in classroom reading comprehension activities. Int. J. New Technol. Res, 5(7), 39. DOI:10.31871/IJNTR.5.7.6.

29. Dizon, G. (2017). Using intelligent personal assistants for second language learning: A case study of Alexa. Tesol Journal, 8(4), 811830. DOI: $10.1002 /$ tesj. 353 .

30. Dizon, G. (2020). Evaluating intelligent personal assistants for L2 listening and speaking development. Language Learning and Technology, 24(1), 16-26.

31. Pal, D., Arpnikanondt, C., Funilkul, S., \& Varadarajan, V. (2019). User experience with smart voice assistants: the accent perspective. In: 10th International Conference on Computing, Communication and Networking Technologies (ICCCNT), pp. 1-6. DOI: 10.1109/ICCCNT45670.2019.8944754.

32. David, B., Chalon, R., Zhang, B., \& Yin, C. (2019). Design of a collaborative learning environment integrating emotions and virtual assistants (chatbots). In: 2019 IEEE 23rd International Conference on Computer Supported Cooperative Work in Design (CSCWD), pp. 51-56. DOI: 10.1109/CSCWD.2019.8791893.

33. Butler, L. (2020). "HEY GOOGLE, HELP ME LEARN" Voice Assistant Devices in the New Zealand Primary School (Doctoral dissertation).

34. Dousay, T. A., Hall, C. (2018). Alexa, tell me about using a virtual assistant in the classroom. In: EdMedia + Innovate Learning, pp. 1413-1419.

35. Schoegler, P., Ebner, M., \& Ebner, M. (2020). The Use of Alexa for Mass Education. In: EdMedia+ Innovate Learning, pp. 721-730.

36. Hales, P. D., Anderson, M., Christianson, T., Gaspar, A., Meyer, B. J., Nelson, B., ... \& Vande Weerd, M. (2019). Alexa?: Possibilities of Voice Assistant Technology and Artificial Intelligence in the Classroom. Empowering Research for Educators, 3(1), 4.

37. Van Brummelen, J. (2019). Conversational Artificial Intelligence Development Tools for K-12 Education. In: 2019 Symposium on AAAI.

38. Bidarra, J., Holmes, W., \& Køhler Simonsen, H. (2020). Artificial Intelligence in Teaching (AIT): A road map for future 
Thanasis Tsourakas, George Terzopoulos* and Stefanos Goumas/Journal of Engineering Science and Technology Review 14 (4) (2021) 1 - 9

developments. In: Empower EADTU, Webinar week: Artificial Intelligence in Online Education. DOI 10.13140/RG.2.2.25824.51207.

39. Winkler, R., Bittner, E., Söllner, M. (2019b). Alexa, Can You Help Me Solve That Problem?: Understanding the Value of Smart Personal Assistants as Tutors for Complex Problem Tasks. In: 14th International Conference on Wirtschaftsinformatik, pp. 371-376.

40. Winkler, R., Neuweiler, M. L., Bittner, E. A., Söllner, M. (2019a). Hey alexa, please help us solve this problem! How interactions with smart personal assistants improve group performance. In: 14th International Conference on Information Systems, pp. 1-17.

41. Bortoli, M., Furini, M., Mirri, S., Montangero, M., \& Prandi, C. (2020). Conversational interfaces for a smart campus: A case study. In Proceedings of the International Conference on Advanced Visual Interfaces, pp. 1-5. DOI: 10.1145/3399715.3399914.

42. Ondáš, S., Pleva, M., \& Hládek, D. (2019). How chatbots can be involved in the education process. In: 2019 17th International Conference on Emerging eLearning Technologies and Applications (ICETA), pp. 575-580. DOI:10.1109/ICETA48886.2019.9040095.

43. Ochoa-Orihuel, J., Marticorena-Sánchez, R., \& Sáiz-Manzanares, M. C. (2020). Moodle LMS Integration with Amazon Alexa: A Practical Experience. Applied Sciences, 10(19), 6859. DOI:10.3390/app10196859.

44. Bakhai, A., Constantin, A., \& Alexandru, C. A. (2020). Motivateme!: An Alexa skill to support higher education students with autism. In: International Conferences Interfaces and Human Computer Interaction, pp. 187-191.

45. Sáiz-Manzanares, M. C., Marticorena-Sánchez, R., \& OchoaOrihuel, J. (2020). Effectiveness of using voice assistants in learning: A study at the time of COVID-19. International journal of environmental research and public health,17(15), 5618. DOI: 10.3390/ijerph17155618.

46. Zhao, J., Bhatt, S., Thille, C., Zimmaro, D., Gattani, N., \& Walker, J. (2020). Introducing Alexa for E-learning. In: Proceedings of the Seventh ACM Conference on Learning@ Scale, pp. 427-428. DOI: 10.1145/3386527.3406719.

47. Emerling, C. R., Yang, S., Carter, R. A., Zhang, L., \& Hunt, T. (2020). Using Amazon Alexa as an Instructional Tool During Remote Teaching. TEACHING Exceptional Children, 53(2), 164167. DOI:10.1177/0040059920964719.

48. Babic, S., Orehovacki, T., \& Etinger, D. (2018). Perceived user experience and performance of intelligent personal assistants employed in higher education settings. In: 2018 41st International Convention on Information and Communication Technology, Electronics and Microelectronics (MIPRO), pp. 830-834. DOI: 10.23919/MIPRO.2018.8400153.

49. Festerling, J., \& Siraj, I. (2020). Alexa, what are you? Exploring primary school children's ontological perceptions of digital voice assistants in open interactions. Human Development, 64(1), 26-43. DOI: $10.1159 / 000508499$.

50. Van Brummelen, J., Tabunshchyk, V., \& Heng, T. (2021). "Alexa, Can I Program You?": Student Perceptions of Conversational Artificial Intelligence Before and After Programming Alexa. In: Interaction Design and Children (IDC '21), pp. 305-313. DOI: $10.1145 / 3459990.3460730$

51. Incerti, F. (2017). Amazon Echo: Emerging technology for formal or informal learning?. In: Society for Information Technology \& Teacher Education International Conference, pp. 1627-1633.

52. Lopatovska, I., \& Oropeza, H. (2018). User interactions with "Alexa" in public academic space. In: Proceedings of the association for information science and technology, pp. 309-318. DOI: $10.1002 /$ pra2.2018.14505501034.

53. Lemmer, S. C. Alexa, Are You Friends With My Kid? Smart Speakers and Children's Privacy Under the GDPR. Smart Speakers and Children's Privacy Under the GDPR. King's College London Law School Graduate Student Research Paper.
54. Lau, J., Zimmerman, B., Schaub, F. (2018). Alexa, are you listening?: Privacy perceptions, concerns and privacy-seeking behaviors with smart speakers. In: Proceedings of the ACM on Human-Computer Interaction, pp. 1-31. DOI: https://doi.org/10.1145/3274371.

55. Liao, Y., Vitak, J., Kumar, P., Zimmer, M., \& Kritikos, K. (2019). Understanding the role of privacy and trust in intelligent personal assistant adoption. In: International Conference on Information, pp. 102-113. DOI:10.1007/978-3-030-15742-5 9.

56. Liao, S., Wilson, C., Cheng, L., Hu, H., \& Deng, H. (2020). Measuring the effectiveness of privacy policies for voice assistant applications. In: Annual Computer Security Applications Conference, pp. 856-869. DOI: 10.1145/3427228.3427250.

57. Loideain, N. N., \& Adams, R. (2020). From Alexa to Siri and the GDPR: the gendering of virtual personal assistants and the role of data protection impact assessments. Computer Law \& Security Review, 36. DOI: 10.1016/j.clsr.2019.105366.

58. Manikonda, L., Deotale, A., \& Kambhampati, S. (2018). What's up with privacy? User preferences and privacy concerns in intelligent personal assistants. In: Proceedings of the 2018 AAAI/ACM Conference on AI, Ethics, and Society, pp. 229-235. DOI: $10.1145 / 3278721.3278773$

59. Pal, D., Arpnikanondt, C., Razzaque, M. A., \& Funilkul, S. (2020). To trust or not-trust: Privacy issues with voice assistants. IT Professional, 22(5), 46-53. DOI: 10.1109/MITP.2019.2958914.

60. Malkin, N., Deatrick, J., Tong, A., Wijesekera, P., Egelman, S., Wagner, D. (2019). Privacy Attitudes of Smart Speaker Users. In: Proceedings on Privacy Enhancing Technologies, pp. 250-271. DOI:10.2478/popets-2019-0068.

61. Cho, E., Sundar, S. S., Abdullah, S., \& Motalebi, N. (2020). Will deleting history make alexa more trustworthy? effects of privacy and content customization on user experience of smart speakers. In: Proceedings of the 2020 CHI Conference on Human Factors in Computing Systems, pp. 1-13. DOI: 10.1145/3313831.3376551.

62. Chalhoub, G., \& Flechais, I. (2020). "Alexa, are you spying on me?": Exploring the Effect of User Experience on the Security and Privacy of Smart Speaker Users. In: HCI for Cybersecurity, Privacy and Trust. HCII, pp. 305-325. DOI: 10.1007/978-3-030-50309321.

63. Lèi, X., Tu, G. H., Liu, A. X., Ali, K., Li, C. Y., \& Xie, T. (2017). The Insecurity of Home Digital Voice Assistants - Vulnerabilities, Attacks and Countermeasures, In: 2018 IEEE Conference on Communications and Network Security (CNS), pp. 1-9, DOI: 10.1109/CNS.2018.8433167.

64. Castell-Uroz, I., Marrugat-Plaza, X., Solé-Pareta, J., \& Barlet-Ros, P. (2019). A first look into Alexa's interaction security. In: Proceedings of the 15th International Conference on emerging Networking EXperiments and Technologies, pp. 4-6. DOI: $10.1145 / 3360468.3366769$.

65. Sharif, K., \& Tenbergen, B. (2020). Smart Home Voice Assistants: A Literature Survey of User Privacy and Security Vulnerabilities. Complex Systems Informatics and Modeling Quarterly, 24, 15-30. DOI:10.7250/csimq.2020-24.02.

66. Chung, H., Iorga, M., Voas, J., \& Lee, S. (2017). Alexa, can I trust you?. Computer, 50(9), 100-104. DOI: 10.1109/MC.2017.3571053.

67. Zhang, N., Mi, X., Feng, X., Wang, X., Tian, Y., Qian, F. (2019). Dangerous skills: Understanding and mitigating security risks of voice-controlled third-party functions on virtual personal assistant systems. In: 2019 IEEE Symposium on Security and Privacy (SP), pp. 1381-1396.

68. Feng, H., Fawaz, K., \& Shin, K. G. (2017). Continuous authentication for voice assistants. In Proceedings of the $23 \mathrm{rd}$ Annual International Conference on Mobile Computing and Networking, pp. 343-355. DOI: 10.1145/3117811.3117823.

69. Alhadlaq, A., Tang, J., Almaymoni, M., \& Korolova, A. (2017). Privacy in the Amazon Alexa skills ecosystem. Star, 217(11). 